\title{
"COSİ, VESTIVA IN BARBARI ACCENTI, / IL VERO AFFETTO A UN AUSONIO": SUGLI SCRITTI IN ITALIANO DI P.B. SHELLEY
}

\author{
FRANCESCO ROGNONI (*)
}

SuNTO. - I tentativi di composizione in italiano di P.B. Shelley si collocano tutti tra il tardo autunno del 1820 e l'agosto del 1821: si tratta di alcune auto-traduzioni, alcuni frammenti isolati e l'abbozzo di una composizione poetica originale, una prosa allegorica, il resoconto di una "improvvisazione" classica di Tommaso Sgricci e alcune lettere più o meno frammentarie. Che significato hanno questi scritti nel complesso dell'opera di Shelley? Che genere di padronanza dell'italiano suggeriscono? E qual è la loro portata espressiva? Come è più opportuno presentarli in una moderna edizione dell'opera shelleyana? La comunicazione cerca di rispondere a questi interrogativi, suggerendo di non assegnare a questi scritti italiani un peso eccessivo, ma neppure di ignorarli del tutto, e concludendo che la loro rilevanza è piuttosto biografica (ci dicono qualcosa su Shelley) che letteraria o linguistica.

$$
* * *
$$

AвSTRACT. - P.B. Shelley's attempts at writing in Italian date to the period from late Autumn 1820 to August 1821. They consist of self-translations, loose fragments, the draft of an original verse composition, an allegorical prose piece, the review of an "improvvisazione" on a classical subject by Tommaso Sgricci, and some more or less fragmentary letters. What is the value of these writings in relation to Shelley's works? What knowledge of Italian do they suggest? What is their expressive import? How can they be best presented in a modern edition of Shelley's works? In addressing these questions, I will suggest that these writings should be neither overvalued, nor completely ignored, and I will conclude that they have a biographical, rather than a literary, or linguistic, relevance.

${ }^{(*)}$ Università Cattolica di Milano, Italia. E-mail: francesco.rognoni@unicatt.it 
Ci si provi a immaginare P.B. Shelley, in uno di quei periodi - in verità, beato lui!, piuttosto brevi - in cui, scoprendosi incapace di composizione originale, si rivolgeva alla traduzione per non perder la mano e, nel frattempo, esplorare nuovi territori. E Dante è sempre un territorio nuovo. In Inghilterra Shelley ne aveva tradotto il famoso sonetto a Cavalcanti, "Guido, io vorrei, che tu, e Lappo, ed io", incluso nel suo libro del 1816, Alastor; Or, the Spirit of Solitude: and Other Poems, e in Italia, nella primavera-estate del '20, aveva reso in terza rima parte del canto di Matelda. Ora, presumibilmente a Pisa, nell'autunno-inverno del '20-'21, sta traducendo la prima canzone del Convito, "Voi, che "ntendendo, il terzo Ciel movete": dove quel "ntendendo nel primo verso gli sta dando un certo filo da torcere, non convincendolo né "Ye who by an intelligent" né "Ye who are designing", soluzioni entrambe cassate nel manoscritto (Bodleian MS. Shelley adds. e. 8, pp. 167-8 rev.):" così che la poesia appare, negli incipitari delle moderne edizioni, come Ye who [ ] the third Heaven move. La difficoltà del primo verso non gli ha comunque impedito di procedere nella traduzione. È già arrivato nel mezzo della quarta strofe, l'ultima prima del congedo, dove "uno spiritel d'amor gentile", rivolgendosi direttamente alla sua "Anima", che teme di esser stata uccisa da Amore, la rassicura che lei (l'anima) non è "morta", ma semplicemente non riconosce più la propria vita, tanto è stata trasformata:

Che quella bella donna, che tu senti, Ha trasformata in tanto la sua vita, Che n'hai paura, sì se' fatta vile.

For that fair lady whom thou dost regret

Hath so [transformed] the life that thou once led,

Thou fearest it - so worthless art thou made -

Così Shelley aveva tradotto impeccabilmente in prima battuta, seguendo la logica (la difficile logica poetico-argomentativa di Dante!) e il proprio istinto di poeta: salvo cassare «that thou» e sostituirvi l'attuale lezione - "the life which once she led" - per fedeltà al testo dell'edizione che possedeva, Delle opere di Dante Alighieri con le annotazioni del dottore Anton Maria Biscioni (Venezia, Gatti 1793), che legge sua

1 Adamson 1992, pp. 430-3. 
invece di «tua vita» (evidentemente per una svista del tipografo, dato che altre edizioni annotate dal Biscioni, ad es. Pasquali, Venezia 1741, sono corrette).

Questo vignetta può insegnare qualcosa sullo Shelley traduttore dall'italiano (e non solo): che affronta e restituisce il testo con piglio e intelligenza di poeta, ne rilegge l'originale con diligenza e rispetto, e corregge la propria traduzione di conseguenza, ma senza prendersi l'ulteriore briga di rileggersi per assicurarsi che tutto sia a posto, nel dubbio - Shelley non ignora che i tipografi lasciano un sacco di errori, nelle sue lettere si lamenta spesso dei refusi e peggio che trova nei suoi stessi libri, in particolare Prometheus Unbound - nel dubbio, dicevo, ricorrendo a un'altra edizione di Dante, che non gli sarebbe certo stato difficile procurarsi anche a Pisa.

A detta del cugino Thomas Medwin, che frequentò Shelley in Italia, e per primo pubblicò una sua composizione italiana, la lirica cortese Buona Notte, Shelley "non aveva [mai] approfondito lo studio dell'italiano, che, come lo spagnolo, aveva acquisito senza una grammatica, fidando nel proprio orecchio fino e nella memoria, più che nelle regole". ${ }^{2}$ Questo senza dubbio fu il metodo di Shelley per tutte le lingue moderne con cui sviluppò una certa dimestichezza (il francese, lo spagnolo appunto, il tedesco). E anche, in certa misura, con le lingue classiche: se è vero che a Thomas Jefferson Hogg - il quale, ancora nel novembre 1817, faceva notare con riprovazione che il lessico greco-latino dello Scapula non era mai entrato a far parte della biblioteca dell'amico - Shelley spiegava di "non aver ancora trovato il coraggio di accettare lo Scapula come mio mentore e guida attraverso i pergolati del piacere del greco", nel rischio "di perdere il fine [cioè, il piacere] in un eccesso di preoccupazione per i mezzi [il lessico]; e di sostituire gli abbracci di una Calipso viva e tangibile, con l'immagine di una Penelope che, benché saggia, non potrà mai ringiovanire".

Non sappiamo esattamente quando Shelley abbia provato a leggere in italiano (una lingua ai tempi assai amata dagli inglesi, che diede di che vivere a molti nostri esuli), né se avesse, almeno quella prima volta, dizionario e grammatica a portata di mano. Improbabile che ne facesse grande uso, quando ricomincerà ad impararlo, leggendo il Beccaria (ma anche,

2 Medwin 1913, p. 351.

3 Shelley 1964, I, p. 569. 
sembra, Tasso e Petrarca) con l'aiuto di Cornelia Turner. "Non t'avevo detto", scriverà in quell'occasione, il 16 marzo 1814, sempre a Hogg, "che pensavo che [Cornelia] fosse fredda e riservata? Ebbene, è esattamente il contrario, come è il contrario di ogni cosa brutta. Ha ereditato tutta la divinità di sua madre" ${ }^{4}$ - la signora Boinville, affascinante vedova di un emigré amico di Lafayette. Da queste due donne, conosciute tramite il futuro suocero, il filosofo William Godwin, Shelley era andato a farsi "coccolare", quell'inverno a Bracknell (nel Berkshire), sbrigliando platonicamente la sua immaginazione erotico-sentimentale, ormai insoddisfatta dalla giovane prima moglie, Harriet Westbrook: ricevendo, quindi, le sue seconde (a sentir lui) lezioni di italiano in un contesto dove, seppur gli abbracci saranno stati esclusivamente linguistici, la saggia "Penelope" del momento era altrettanto attraente della vivace e (castamente) tangibile "Calipso" che gli sedeva vicino e compitava i versi con lui.

C'è una indubbia connessione tra curiosità - diciamo pure, avventurosità - linguistica e immaginazione erotica, che rende tanto più ironica e frustrante la delusione quasi adolescenziale con cui, quando finalmente arriverà in Italia, Shelley si troverà ad occhieggiare le stesse donne che entusiasmavano sui contemporanei più eroticamente adulti, come Byron o Stendhal.

Le donne [italiane] soprattutto sembrano una razza molto inferiore. I loro modi, per quanto possa giudicare dai loro atteggiamenti e dalla loro fisionomia, sono puritani e civettuoli al tempo stesso; i loro volti sono scarni; i corpi sottili, e quelle che hanno qualche pretesa di essere belle possiedono una bellezza superficiale, fredda e arida. La loro voce, che non ha proprio l'accattivante dolcezza delle Francesi, è dura, e priva di inflessione e varietà di tono. Ma queste considerazioni, per quanto ne so, valgono solo per Milano, ché la mia esperienza non si spinge oltre.

Ma ahimè, anche spingendosi oltre, Shelley sembra non incontrare niente di meglio: "Le donne sono particolarmente vuote, $[\ldots]$ prive di qualsiasi cultura e raffinatezza", scrive il 25 luglio 1818 al suocero William Godwin; il che (che fosse il suocero) potrebbe anche significare il contrario, non fosse che l'idea è ribadita, lo stesso giorno, all'amico scapolo Thomas Love Peacock: "Le donne [di Bagni di Lucca] sono ben lontane da qualsiasi cosa l'osservatore più indulgente potrebbe

4 Shelley 1964, I, p. 384. 
chiamare bellezza o grazia, e non sembrano possedere alcuna intelligenza particolare che compensi questa mancanza”. Per non parlare delle veneziane che - altra lettera a Peacock, dicembre dello stesso anno, "sono forse le più spregevoli sotto la luna: le più ignoranti, le più disgustose, le più bigotte, le più sporche. Le contesse puzzano talmente di aglio che un inglese normale non può neanche avvicinarsi".

Può quindi darsi che non sia soltanto una questione di tempi (Shelley viveva in Italia ormai da più di due anni) se quasi tutte le prove in italiano di Shelley risalgono al tardo autunno-inverno 1820-21 e sono più o meno direttamente riconducibili alla frequentazione della bella Teresa Viviani, "l'unica Italiana per la quale abbia mai provato qualche interesse". ${ }^{6}$ Si tratta di due prose di un certo impegno, una favoletta allegorica (che preannuncia Epipsychidion, e almeno un momento di The Triumph of Life) e il resoconto di una improvvisazione di Tommaso Sgricci (sorta di "palestra" per vari passi della Defence of Poetry, soprattutto quelli dove viene discussa l'irrazionalità dell'ispirazione); alcuni abbozzi di lettere (in uno dei quali è citato il sonetto di Dante a Cavalcanti già ricordato); un gruppetto di auto-traduzioni; la rielaborazione in italiano di una poesia già scritta in inglese; qualche breve frammento e almeno un tentativo abbastanza prolungato (ci restano circa trenta versi) di composizione poetica originale.

Su chi fosse Teresa Viviani, lascio la parola a Mary Shelley, che l'introduce all'amico di famiglia Leigh Hunt nella sua lettera in (non cattivo) italiano del 3 dicembre 1820:

V'è un'altra conoscenza nostra romanesca e patetica e una fanciulla di diece novi anni - figlia d'un nobile Fiorentino, bellissima - d'un gran' genio - chi scrive Italiana con un eleganza e delicatezza chi eguala i migliore autori della migliore età d'Italia - Ma è infelicissima - La sua madre è una pessima donna: e essendo gelosa dai talenti e la belezza della di sua figlia, la rinchiude in convento dove non vede mai che le cameriere e le idiote. Ne esce mai ma chiusa in due piccole stanze che guardono - sulla kitchen gar[den] poco pittoresco del convento, lamenta sempre la sua pietosa condizione - La sola sua speranza è di maritarsi Ma la sua essistenza stessa e quasi un secreto - e che sposaliza rara! ${ }^{7}$

5 Shelley 1995, pp. 1098, 1104, 1105 e 1135.

6 Shelley 1995, pp. 1236.

7 Shelley 1980, I, p. 163. 
Nella stessa lettera, Mary racconta a Hunt, con un bel gusto per il dettaglio, anche dell'eccentrico professor Pacchiani e di Tommaso Sgricci, l'improvvisatore: perché a Pisa, quell'autunno, gli Shelley, che in Italia finora avevano frequentato soprattutto altri inglesi, cominciano a venire in contatto anche con personaggi locali di un certo spicco (come, non menzionato in questa lettera, un celebre medico, il professor Vaccà). Ci sarà stato un grande esercitarsi in italiano, in quelle settimane (anche la lettera a Hunt è, ovviamente, un esercizio), quasi di certo accompagnato dalla gratificante scoperta che la lingua del sì, che da più di due anni gli Shelley orecchiavano, era ormai quasi pronta a "passare anche alla bocca", a farsi appunto "lingua" - e lingua delle cui imperfezioni non vergognarsi troppo: un po' come Sgricci, che improvvisava le sue tragedie come in nessun'altra nazione si sarebbe osato, non si vergognava punto dei loro difetti, badando solo a trascinare il pubblico. Del resto, non era la Corinne di Madame de Staël, sinonimo dell'Italia, proprio una "improvvisatrice"?

All'inizio, quella per la bella reclusa sembra esser stata una passione collettiva. Non solo Shelley, ma anche Mary e sua sorella acquisita, Claire Clairmont, le facevano visita in convento. Fra le giovani donne era un via vai di regalini. Ci si scambiava lezioni di lingua straniera e s'inventavano nomignoli; o ci si rivolgeva l'un l'altra, familiarmente (e biblicamente), come "fratello" e "sorella". Poi la fantasia erotico-sentimentale di Shelley deve essersi accesa un po' più del dovuto, forse riscaldandosi - prima d'avvampare nella gran fiammata dell'Epipsychidion - proprio grazie all'esercizio d'auto-traduzione di quella che ha tutto l'aspetto di una breve antologia tematica della sua opera: una sorta di biglietto di presentazione, o carta di identità, dove le quattro strofi del Laon and Cythna (i versi 667-98) costituisco un autoritratto idealizzato (la foto!), la Ode to Liberty (tradotta integralmente) enuncia il credo politico-libertario, e le liriche dal Prometheus Unbound (II, v 48-110 e IV 1-82) dichiarano l'amore e il suo afflato universale. Anche il nome con cui Shelley si rivolgeva a Teresa, e con cui la indica nell'Epipsychidion, è stato probabilmente "trovato in traduzione": solo recentemente, ${ }^{8}$ infatti, si è riconosciuto che quello che finora era considerato il frammento di una lettera, è in realtà la resa in zoppicanti endecasillabi italiani ("Che Emilia, ch'era più bella [a vedere] /

$8 \quad$ Shelley 2014, pp. 52-53. 
Che il giglio bianco sul suo verde stelo / E piu fresca che la Maia quando") di un passo del Knight's Tale di Chaucer, a sua volta derivato dal Teseida del Boccaccio - dove l'eroina, Emilia appunto, è contesa tra due spasimanti come sembra fosse il caso di Teresa Viviani.

Una certa attenzione critico-biografica e linguistica, questi testi l'han ricevuta.' La domanda cui, in tempi recenti, m'è stato chiesto di rispondere, è: quanto "seriamente" va preso, l'italiano di Shelley, in una moderna edizione delle sue poesie, come quella - finalmente in via di completamento, a quasi cinquant' anni dalla sua ideazione, a venticinque dall'uscita del primo volume - dei "Longman Annotated English Poets"? Abbastanza ma non troppo, direi; anche perché altrimenti si rischia di farsi sfuggire l'aspetto, non solo galante, ma anche ludico dell'esperimento, restando alla fine solo con le ceneri dell'auto-compiacimento (che è, senza dubbio, un altro degli aspetti della faccenda). E qui colgo il pretesto per riconoscere un bel po' di responsabilità nelle scelte, implicite ed esplicite, del quarto volume, fresco di stampa (novembre 2013), dell'edizione Longman (ora Routledge), dove i testi italiani vengono emendati solo ove strettamente necessario, ed annotati con la massima economia, resistendo all'ingenua, ingenerosa tentazione di correggere o modernizzare in nota l'italiano di Shelley. Ho invece suggerito ai curatori, Michael Rossington, Jack Donovan e Kelvin Everest, che si integrassero apostrofi e accenti (discostandomi, in questo, dal criterio seguito, ad esempio, da Betty T. Bennett nello stabilire il testo della lettera di Mary Shelley citata sopra), anche perché è impossibile dire dove Shelley li ometta di proposito (cioè per errore!) e dove semplicemente - come spesso avviene anche per la punteggiatura dei manoscritti inglesi - la sua penna corra troppo velocemente sul foglio. Infine, trattandosi di una collana di alto rigore filologico, ma che prevede la modernizzazione di arcaismi ortografici e convenzioni tipografiche, ho suggerito di adottare la convenzione moderna di distinguere fra accento grave e accento acuto sulla "e", anche se, senza dubbio, Shelley non ebbe mai in mano un libro italiano con accenti anche acuti. È una scelta, questa, improntata a uno spirito di massima praticità, che ha seguito anche Valentina Varinelli, stabilendo il testo dei versi italiani di Shelley in appendice all'edizione rivista ed accresciuta delle Opere a

9 Vedi, ad es., Koszul 1922, De Palacio 1975, Shelley 1995, pp. 1805-08, Capelli 2009, Shelley 2014, passim. 
mia cura (Einaudi, Biblioteca della Pléiade 1995), che uscirà, in due volumi, nella collana dei Meridiani Mondadori nel 2018.

Tornando alle poesie: non sembra che Shelley sia mai giunto allo stadio di "amare" l'italiano al punto di degustarne certi vocaboli del parlato, come fa Byron con la diletta seccatura ("a devilish good word", la definisce, nella lettera a T. Moore del 6 novembre 1816$)^{10} \mathrm{o}$ - sorprendentemente - Stendhal con la parola fiasco (cui dedica un capitoletto dei Souvenirs d'égotisme; dove - sia detto per inciso - Beyle attribuisce a Shelley, di cui si diceva amico pur non avendolo mai incontrato, un'epigrafe tratta in realtà da La vie de Marianne di Marivaux $\left.{ }^{11}\right)$. Gli Shelley naturalmente si rendevano conto che la lingua che ascoltavano ogni giorno era ben diversa da quella nobile della letteratura (Mary, nella lettera già citata a Hunt [3 dicembre 1821], scrive, del "diavolo" Pacchiani, che "Parla una bellissima lingua Italiana, tutto differente della idioma di oggi, che ci fa credere d'udire il Boccacio o il Macchiavelli parlando come scrissono"12). Ma è difficile dire quanto Shelley fosse consapevole che lingua della poesia e lingua parlata, in Italia ai suoi tempi (e non solo ai suoi tempi, ma fino a una cinquantina d'anni fa, se non meno ancora), erano molto più distanti di quanto lo siano sempre state nella cultura inglese, anche da molto prima della "rivoluzione" delle Lyrical Ballads (dove, appunto, si dichiarava che "i Poeti non scrivono solo per i Poeti, ma per gli uomini", perciò sono invitati "ad esprimersi come tutti gli altri", a sceglierei le proprie parole "dalla vera lingua degli uomini").

Vagliare gli errori in cui Shelley incorre più di frequente, segnalare le molte scempie, gli articoli omessi (soprattutto davanti al possessivo), la mancata concordanza di aggettivo e sostantivo, i cosiddetti "falsi amici”, ecc. è sicuramente meno interessante che indicare certe occasio-

10 Byron 1976, p. 125.

11 In ben due testamenti, quello del 10 e 11 dicembre 1832 e quello del 22 e 28 maggio 1834, Stendhal chiede d'esser sepolto a Roma nel cimitero degli inglesi, "près de mon ami Shelley (pyramide de Cestius)" (Stendhal 1982, p. 999). Fra il romanziere francese e il poeta inglese non risulta però nessun incontro: certo non quello che, stando a Stendhal (Rome, Naples et Florence, 29 dicembre 1816), sarebbe avvenuto a Bologna nel dicembre del '16, data alla quale Shelley si trovava in Inghilterra; anche se è vero che quell'autunno, a Milano, Stendhal aveva incontrato Byron - e questi, che aveva trascorso parte dell'estate in compagnia di Shelley, può avergliene parlato.

12 Shelley 1980, p. 163. 
nali felicità o guizzi espressivi. E poi, come ho già accennato, è difficile dire dove Shelley sbagli effettivamente e dove invece segua usi particolari, locali: ad esempio, nella traduzione del canto degli spiriti del quarto atto del Prometheus Unbound, non è detto che il finale in -ono di "ballono" e "cantono" (vv. 46 e 48) sia necessariamente uno svarione, e non piuttosto la trascrizione di una variante (debitamente segnalata dal Rohlfs) del parlato toscano. ${ }^{13}$ Fra le peculiarità lessicali, è interessante segnalare l'uso ricorrente del sostantivo "eremo" per tradurre l'inglese wilderness / wild / waste / desert ("Un paradiso dei eremi [wildernesses]"; "Per le notturne valli del eremo [desert] anno"; "un eremo [waste] dei flutti"; "Per l'eremo [wild] che sente nulla"). ${ }^{14}$ Almeno in un paio di casi, l'immagine tradotta sembra acquistare vigore e concretezza rispetto all'originale: in Laon and Cythna, ad esempio, Shelley scriveva astrattamente di "Victims who worshipped ruin" (v. 689) mentre in italiano l'immagine si personalizza in modo direi quasi sadico, diventando: "vittime che adarovono il sacrificatore". E anche l'inizio della strofe successiva, "The land in which I lived, by a fell bane / Was withered up" (vv. 694-95), acquista in traduzione - "La patria mia era marchiata da una / Amara peste" -, con l'uso di "marchiata" invece del più neutro, impersonale "inaridita" (o consimili), una vividezza drammatica assai più forte. E non è escluso che nel caso della seconda strofe della Ode to Liberty, dove Shelley traduce il v. 22, "Was yet a chaos and a curse" con "Era ancora caos e nulla" e, poco sotto (v. 28), svincolato dalle esigenze della rima, opta per "madre" invece di "nurse", la resa italiana sia (come è stato suggerito ${ }^{15}$ semanticamente più felice, e forse nelle più autentiche intenzioni di Shelley: almeno per quel che riguarda la sostituzione di "madre" a "nutrice" (ché, nel sistema poetico di Shelley, la parola "curse", maledizione, anche a prescindere dalla consonanza (chaos / curse), ha una valenza tutta particolare, una carica distruttiva forse ancor più possente del "nulla").

A proposito della Ode to Liberty, da cui provengono questi esempi. È difficile immaginare che Shelley abbia tradotto le diciannove stan-

13 Devo l'osservazione, accolta in una nota a Shelley 2014, p. 20, allo storico della lingua italiana e carissimo amico Antonio Daniele.

14 A rendere rispettivamente Prometheus Unbound II, v 81 e IV 76, e Ode to Liberty 39 e 51.

15 De Palacio 1975, p. 237. 
ze (duecentottantacinque versi) d'una poesia politica di tale complessità solo a edificazione di Teresa; e ancor più difficile pensare che la giovinetta, per quando infatuata e, chiusa in convento com'era, a corto di libertà, fosse disposta a sorbirsela dall'inizio alla fine. Più probabile che a spinger Shelley ad auto-tradursi ci fosse anche dell'altro. Forse, come suggerisce Michael Rossington nel commento dell'edizione Longman, la speranza era che la poesia, se pubblicata su un giornale italiano, potesse influenzare l'opinione pubblica a favore del nuovo governo costituzionale napoletano, o più generalmente all'indipendenza dall'Austria. Con la ripulita di un letterato italiano (o forse anche solo di Teresa), l'ode sarebbe potuta uscire, ad esempio, sull'appena inaugurata "Antologia" del Vieusseux, che nel primo numero (gennaio 1821) includeva la traduzione di Michele Leoni di una poesia di Lamartine dedicata a Byron. Il quale Byron, che di regola non amava che le sue poesie fossero tradotte (in un caso aveva addirittura pagato un poeta nostrano perché rinunciasse a farlo), sempre nel '21 si sarebbe compiaciuto che Lorenzo Da Ponte, il librettista di Mozart, avesse tradotto e pubblicato a New York la sua Prophecy of Dante, "intesa per gli italiani e per la Guiccioli" ${ }^{16}$ ma troppo antiaustriaca per uscire anche in Italia (dove pure, probabilmente all'insaputa di Byron, anche Michele Leoni ne aveva fatto una traduzione). Se qualcosa di simile è vero anche per la Ode to Liberty, è un'ironia della sorte che il manoscritto dell'auto-traduzione sia giunto a noi senza il fascicolo con le strofe 14-18 (abbiamo le prime tredici e l'ultima, la diciannovesima), quindi ci manchi anche l'esortazione più diretta agli italiani: "O Italy, / Gather thy blood into thy heart; repress / The beasts who make their dens thy sacred palaces" (vv. 208-10).

L'Ode alla Libertà fornisce diversi esempi di quegli epiteti composti - come "terra-svegliante", "spirito-alata", "spirito-instinta", "Aurora-illuminato" -, prediletti da Shelley, che sono uno dei tanti motivi di scoramento del traduttore. Che l'italiano non consenta queste composizioni, lo "sentiva" anche Shelley, che in qualche caso riesce ad evitarle (rendendo, ad esempio, "all-sustaining air" [v. 20] con "aria che sostiene tutto"). Anche se una loro "accettabilità" poetica, forse, questi "echi di stilemi omerici" ${ }^{17}$ se la sono quasi guadagnata nella "tradizio-

16 Medwin 1966, p. 159.

17 Papetti 1988, p. 26. 
ne" delle traduzioni: vi fa un certo ricorso, ad esempio, una fine traduttrice come Viola Papetti nella sua versione dell'Endymion di Keats (anch'io mi sono concesso un "elisee isole-giardino" traducendo 1' "Elysian garden islets" di Prometheus Unbound II, v 91 - che Shelley, nella sua autotraduzione, semplifica in "Elisee isolette"). Degno d'attenzione il penultimo degli esempi citati: con l'espressione "[faccia] spirito-instinta" Shelley si sforza di tradurre "spirit-sighted [countenance]" (v. 148), detta del volto di Milton, cieco e tuttavia che non trasmette l'impressione della cecità, ma della vista interiore, spirituale. "Instinct" è un classico "falso amico". Un tentativo di poesia italiana originale, esattamente coevo all'auto-traduzione dell'Ode alla Libertà, inizia: "Dal spiro della tua mente, istinta / La chiara fronte...", dove Shelley ovviamente intende "permeata", "animata" (come nel pezzo dedicato a Tommaso Sgricci, in cui si afferma che "Suoi gesti [si noti l'omissione dell'articolo davanti al possessivo!] il tuono della sua voce, il suo viso, tutto istinto dalla vita della ispirazione"). ${ }^{18}$ La splendida attenzione critico-filologica che questo frammento ha ricevuto da parte di Donald Reiman, il decano dei moderni studi shelleyani, promotore di svariate iniziative editoriali, non ha impedito che, nel commento alla sua edizione diplomatica del manoscritto, il primo verso venga frainteso, e risulti tradotto, insensatamente: "From the breath [exhalation] of thy mind [is] intuited / The bright brow...". ${ }^{19}$ Né Reiman né i due esperti di italiano da lui consultati hanno, evidentemente, colto che "istinta" non è che un calco di "instinct".

V'è un passo della Defence of Poetry in cui Shelley afferma che "Livy is instinct with poetry", cioè che la sua prosa è permeata, imbevuta di poesia; e circola (o circolava) una traduzione, pubblicata con contributi ministeriali, che legge "L'arte di Livio è sintesi di istinto e poesia”..! Chi ha pratica di traduzione sa che gli svarioni di questi genere sono praticamente inevitabili, e qui lancio un pietra (pur sapendo d'aver commesso anch'io un'infinità di peccati, anche più gravi, come tocco con mano riaggiustando le mie traduzioni di Shelley per la nuova edizione cui ho già accennato) solo perché mi sembrano più perdonabili - almeno fanno sorridere! - di quelli che ci arrivano paludati d'an-

18 Shelley 1995, p. 1332; corsivo mio.

19 Reiman 1990, pp. 90-91. La traduzione proposta in Shelley 2104, p. 196, è: "By the spirit of your mind is animated / The clear brow...". 
notazioni. Mi è recentemente capitato di esaminare il manoscritto di sei stornelli improvvisati da Byron in italiano per la Guiccioli, pubblicati da Jerome McGann nella sontuosa edizione della Clarendon Press. Eccone il quarto e il sesto:

Tomaso con Giusepe han litigato

il Sior Pierino è stato ferito

ci mancò poco che non fu amazzato-

Fior di viole

il Sior Pierino mi vuole abbandonare

e lascia lo suo amore e lo suo core- ${ }^{20}$

Tutto chiarissimo, e piuttosto divertente (Byron l'italiano lo masticava già ad Atene nel '10, immaginiamoci nel '22, dopo sei anni che viveva in Italia! $\left.!^{21}\right)$. Ma nella trascrizione di McGann, forse per "contagio da stornello", invece di "Sior" si trova "fior Pierino" - un errore marchiano, ripreso dall'eccellente, idiosincratico Peter Cochran nel suo sito web (http://petercochran.wordpress.com/), evidentemente persuaso della dotta nota dell'edizione Clarendon: dove l'improbabile lezione è difesa rimandando a espressioni come "fior di gentiluomo" o consimili, ${ }^{22}$ quando sarebbe probabilmente bastata una telefonata interna al dipartimento di italiano!

Tornando a Shelley. I suoi esercizi in italiano culminano, in inglese!, nell'Epypsichidion, che reca in epigrafe un passo (non tradotto) ${ }^{23}$ dal trattatello platonizzante di Teresa Viviani, Il vero amore. Esplicitamente paragonato alla Vita Nuova (di cui Shelley fu uno dei primissimi lettori inglesi, proprio nel gennaio del '21, si suppone con l'aiuto di Teresa), introdotto dal congedo della prima canzone del Convito (nella traduzione cui ho accennato all'inizio di questo saggio), imbevuto di poesia italiana, il poemetto è il risultato dell'aggregazione di frammenti anche molto antecedenti all'infatuazione di Shelley per la

20 Byron 1991, p. 512.

21 Sull' "impetuoso italiano epistolare di Byron", vedi Brugnolo 2009, p. 39.

22 Lord Byron, The Complete Poetical Works, ed. J.J. McGann, At the Clarendon Press, Oxford 1980-93, vol. 6, p. 512.

23 Giusto notare che le sue epigrafi, per lo più dal greco o dal latino, Shelley non le traduce mai. 
Viviani. Cinquant'anni più tardi, Edward Trelawny sarebbe stato convinto di ricordare che della poesia esisteva anche una versione italiana: una versione originale, precedente quella inglese. Già W.M. Rossetti, cui dobbiamo l'informazione, sospettava che il vecchio avventuriero, per quanto ancora prodigiosamente vigoroso, ricordasse male..$^{24} \mathrm{Ma}$ è probabile che almeno i versi dell'attacco -

Sweet Spirit! Sister of that orphan one,

Whose empire is the name thou weepest on,

In my heart's temple I suspend to thee

These votive wreaths of withered memory.

siano effettivamente stati abbozzati in italiano prima che in inglese. Mi riferisco ai due frammenti indicati, in appendice all'edizione Longman, con le lettere $\mathrm{N}$ e O:

Sul altare del nostro amore, a te, Sorella

Io [offerisco] questi pallidi fiori

Anima dolce, chi sei, la sorella

Di quell'orfana anima che regge

Il nome e la forma mia, bella

(quest'ultimo con allusione all'incipit petrarchesco "Spirto gentil che quelle membra reggi", già notato dal De Bosis nel '13). ${ }^{25}$

Una certa connessione con Epipsychidion presenta, senz'altro, anche il più lungo frammento già ricordato, "Dal spiro della tua mente, istinta". Non solo vi si immagina la navigazione degli amanti verso "Un queto asilo, lontan di ogni pena", come nella poesia inglese sarà verso una "Elysian isle" (sempre 1' "incantamento" del sonetto di Dante a Cavalcanti, citato, s'è detto, anche in un abbozzo di lettera a Teresa!); ma anche la cornice dell'Epipsychidion - che, secondo l'Avvertenza, viene pubblicato postumo da un amico (presumibilmente italiano) dell'autore inglese, morto a Firenze mentre era in procinto di trasferirsi in "una delle più selvagge Sporadi" - sembra anticipata nel frammento di terza rima su cui la poesia italiana si interrompe:

24 Rossetti 1977, p. 169.

25 De Bosis 1913, pp. 14-19. 
Così, vestiva in [barbari] accenti,

Il vero affetto a un [Ausonio]

Tirsi[.]

Che fra le poche poesie giunte fino a noi di Teresa Viviani - versi né più belli né più brutti di quelli di infiniti altri poeti italiani minori di primo ottocento - vi sia Il ritratto di Tirsi, un sonetto che, senza dubbio, traccia un ritratto idealizzato di Shelley, fa pensare che l' "Ausonio" a cui i versi precedenti sono rivolti sia piuttosto una "Ausonia", quell'unica italiana per la quale (parafrasando una lettera già citata) Shelley avesse mai provato interesse.

Chissà se avrebbe continuato a usare l'italiano come lingua della poesia se, quasi ancor prima d'aver dato alle stampe Epipsychidion, non si fosse trovato così disilluso quanto alle vere qualità di Teresa Viviani, che ben presto scoprì essere "una nuvola, non una Giunone" ? ${ }^{26}$ Così il suo unico altro esercizio di italiano che si conosca è dedicato a un'altra Teresa. Si tratta delle due lettere del 10 e 22 agosto 1821, che Shelley scrisse a Teresa Guiccioli, l'amante di Byron, la prima per dissuadere i Gamba, cui era stato ingiunto di lasciare Ravenna, dal trasferirsi a Ginevra, la seconda, poco più di un biglietto, per assicurarli della bella accoglienza che avrebbero ricevuto a Pisa. Finora la lettera del 10 agosto si conosceva solo nella traduzione in francese della stessa Guiccioli. In tempi recenti, il manoscritto originale è stato acquistato dalla Carl $\mathrm{H}$. Pforzheimer Collection, che la darà alle stampe in uno dei prossimi volumi di Shelley and his Circle. Ho avuto la fortuna di prenderne visione. L'italiano di Shelley, in questa lettera, forse stesa con l'aiuto di un quasi madrelingua (come quella del 22 agosto), è piuttosto buono. Qui mi limito a segnalare l'uso disinvolto di una parola efficace come "pretismo", abbastanza attestata fra fine settecento e inizio ottocento (il Tommaseo non la registra, forse per prudenza; ma Garibaldi, in una lettera da Caprera, la definisce da gran lessicografo: "la più scellerata di tutte le piaghe umane"!), di cui la Guiccioli ammorbidisce l'espressività nella sua traduzione-parafrasi - "la victime de l'intolerance du clerge de mon pays"-, quasi un piccolo intervento di censura.

26 Shelley 1995, p. 1307. Il riferimento, nella lettera a John Gisborne del 18 giugno 1822, è al mito di Issione, che pensando di sedurre Era, si accoppiò invece con una nuvola foggiata da Zeus con le sembianze della moglie. 
La lettera termina con un poscritto dove ritornano le stesse metafore dell'ultima terzina di "Dal spiro della tua mente, istinta". Shelley vi si definisce "barbaro" (le lettere centrali della parola sono sottolineate due volte), ovvero non solo straniero, ma goffo, balbuziente, perciò la sua lingua veste, o "vela", i veri affetti o sentimenti che vorrebbe esprimere. E quasi "contro-poesia", se è vero che - come si dichiara nella Defence of Poetry - la poesia, nella sua funzione più alta, non veste bensì denuda: "strappa dal mondo il velo della consuetudine e rivela la nuda bellezza addormentata che è lo spirito delle sue forme" ${ }^{27} \mathrm{E}$ v'è un elemento di imbarazzo, di vergogna, nel suo uso, quasi la stessa "gran vergogna" che - nelle parole della Vita Nuova citate (in italiano senza traduzione) nell'Avvertenza a Epipsychidion "sarebbe a colui, che rimasse cosa sotto veste di figura, o di colore rettorico: e domandato non sapesse denudare le sue parole da cotal veste, in guisa che avessero verace intendimento" (corsivi miei). La lingua straniera è dunque, forse, essa stessa metafora, figura retorica, necessaria e al tempo stesso ostacolo all'espressione. E non è affatto improbabile che la memoria - così fresca eppure forse inconscia - del suo sforzo di poesia italiana sia attiva quando, nell'aprile del '21, nel ritrarre se stesso fra i poeti inglesi che piangono Keats, Shelley canti il suo nuovo dolore in una lingua sconosciuta - "in the accents of an unknown land" (Adonais 301).

\section{BIBLIOGRAFIA}

Adamson C., 1992. The Bodleian Shelley Manuscripts, volume 6, Shelley's Pisan Winter Notebook (1820-1821): Bodleian MS. Shelley adds. e. 8, C. Adamson ed., New York, Garland.

Brugnolo F., 2009. La lingua di cui si vanta amore. Scrittori stranieri in lingua italiana dal Medioevo al Novecento, Roma, Carocci.

Byron, 1976. Byron's Letters \& Journals, volume 5, 1816-1817, L.A. Marchand ed., Cambridge, Mass., Harvard University Press.

Byron, 1991. The Complete Poetical Works, volume 6, J.J. McGann and B. Weller eds., Oxford, At the Clarendon Press.

Capelli R., 2009. Le autotraduzioni di Shelley per amore di Teresa: frammenti di un'autobiografia poetica ideale, in Scrittori stranieri in lingua italiana, dal Cinquecento ad oggi, a cura di F. Brugnolo, Padova, Unipress.

27 Shelley 1995, p. 1042. 
De Bosis A., 1913. On the First Two Lines of "Epipsychidion", "Keats-Shelley Memorial Bulletin”, II, pp. 14-19.

Koszul A., 1922. Inédits italiens de Shelley, «Revue de littérature comparée», II, Juillet 1922, pp. 471-7

Medwin T., 1913. The Life of Percy Bysshe Shelley, H. Buxton Forman ed., London, Oxford University Press.

Medwin T., 1966. Medwin's Conversations of Lord Byron, E.J. Lovell, Jr. ed., Princeton University Press.

Papetti V., 1988. Introduzione a J. Keats, Endimione, Milano, Rizzoli.

Reiman, 1990. The Bodleian Shelley Manuscripts, volume 7, Shelley's Last Notebook: Bodleian MSS. Shelley adds. e. 20, adds. e. 15 and [part of] adds. c. 4, D. Reiman and H. Dworzan Reiman eds., New York, Garland.

Rossetti, 1977. The Diary of W.M. Rossetti, 1870-1873, O. Bonnard ed., Oxford University Press, 1977.

Shelley P.B., 1964. The Letters of Percy Bysshe Shelley, volume 1, F.L. Jones ed., Oxford, At the Clarendon Press.

Shelley P.B., 1980. The Letters of Mary Wollstonecraft Shelley, volume 1, B.T. Bennett ed., Baltimore, The Johns Hopkins University Press.

Shelley P.B., 1995. Opere, a cura di F. Rognoni, Torino, Einaudi (ma si cita dalla nuova traduzione, pronta per l'edizione di Meridiani Mondadori 2018).

Shelley P.B., 2014. The Poems of Shelley, Longman Annotated English Poets, vol. 4, M. Rossington, J. Donovan and K. Everest eds., London, Routledge.

Stendhal, 1982. Oeuvres intimes, volume 2, édition établie par V. del Litto, Bibliothèque de la Pléiade, Paris, Gallimard. 